\title{
On the Relation between Discounting of Climate Change and Edgeworth-Pareto Substitutability
}

\author{
Tomas Kögel \\ Wageningen University
}

\begin{abstract}
To justify substantial carbon emission reductions, recent literature on cost-benefit analysis of climate change suggests discounting environmental quality at a lower discount rate than the standard consumption discount rate. Recent literature also shows that a theoretical foundation for such a lower environmental discount rate requires rising willingness-to-pay for environmental quality (WTP). A widely believed better alternative is however to adjust instead future environmental benefits for rising WTP and to discount those benefits at the consumption discount rate. According to this latter approach, rising WTP is usually assumed not to change the consumption discount rate itself. Assuming environmental resource scarcity, the present paper shows that an unchanged consumption discount rate is however, by and large, only an appropriate assumption in the knife-edge case in which environmental quality and goods consumption are neither substitutes nor complements in the Edgeworth-Pareto sense (substitutes, respectively, complements in the Edgeworth-Pareto sense implies the marginal utility of goods consumption to be decreasing, respectively, increasing in environmental quality).
\end{abstract}

Special issue "Discounting the Long-Run Future and Sustainable Development"

JEL: H43, Q51, Q54

Keywords: Discounting; cost-benefit analysis; climate change

\section{Correspondence}

Tomas Kögel, Wageningen University, Department of Social Science, Environmental Economics and Natural Resources Group, PO Box 8130, 6700 EW Wageningen, The Netherlands, Tel.: +31 317 483318, e-mail: tomas.kogel@wur.nl

Hans-Peter Weikard and Xueqin Zhu is gratefully acknowledged for stimulating discussions. In addition, the paper benefitted from comments by Richard Tol, Reyer Gerlagh and two anonymous referees. 


\section{$1 \quad$ Introduction}

In an effort to mitigate climate change, as of 2008, 183 countries had signed and ratified the Kyoto Protocol, committing to reduce their emissions of carbon. Many people believe that the emission reductions of the Kyoto Protocol are not strong enough. Nevertheless, standard economic cost-benefit analysis has difficulties in justifying the emission reductions of the Kyoto Protocol, not to speak about even higher emission reductions. The main reason for the justification problem is the discount rate. The costs of emission reductions are to be paid now, while the mitigation of damages from climate change will be enjoyed in the future and therefore proper cost-benefit analysis requires to discount them. However, the relevant time horizon for climate change damages is hundred years and more. For this reason, standard discount rates lead to dramatic reductions of the present value of damage mitigation and therefore standard cost-benefit analysis hardly ever suggests substantial emission reductions (see e.g. Nordhaus and Yang, 1996, and Nordhaus and Boyer, 2000). Many environmentally informed researchers are dissatisfied with this conclusion. They believe that standard cost-benefit analysis has to be modified to escape the justification problem of substantial emission reductions. One way out of this dilemma has been suggested in the very influential "Stern Review of the Economics of Climate Change" for the British government, released on 30 October 2006, arguing that ethical considerations call for a very low discount rate. However, the justification of a very low discount rate with ethical considerations has been heavily criticised by many economists as being paternalistic. ${ }^{1}$

Earlier, Yang (2003), assuming a model with utility from goods consumption and environmental quality, proposed an alternative justification for the emission reductions of the Kyoto Protocol, namely dual-rate discounting. More specifically, he suggests that environmental quality should be discounted at an environmental discount rate that is lower than the consumption discount rate, which is used for goods consumption. This is important because standard economic literature usually uses for the value of the consumption discount rate the observed time-invariant market rate of return to capital. An environmental discount rate could however be substantially lower than this market rate. Applying dual-rate discounting to the RICE model of Nordhaus and Yang (1996) and Nordhaus and Boyer (2000), the author confirms that dual-rate discounting can justify the Kyoto Protocol obligations. However, Tol (2004), though expressing sympathy with Yang's reasoning, has an objection on Yang's methodology. More specifically, Tol objects that Yang ensures the environmental discount rate to fall short of the consumption discount rate by assuming different utility discount rates for environmental quality and goods consumption (the former being lower than the latter). As a better alternative, he suggests a model in which income growth might increase the willingness-to-pay for environmental quality (WTP) and in which this rising WTP is reflected in parameter shifts towards a lower environmental discount rate. ${ }^{2}$

The crucial difference between the model assumption of Yang (2003) and the model assumption of Tol (2004) is that dual-rate discounting is required for modelling environmental concerns according to Yang's assumption, while modelling it according

\footnotetext{
${ }^{1}$ Among the critics are Nordhaus (2007), Yohe and Tol (2007), and Weitzman (2007).

${ }^{2}$ See for a similar reasoning Hasselmann et al. (1997) and Horowitz (2002).
} 
to Tol's assumption allows alternatively to adjust future environmental benefits for rising WTP and to discount those benefits at the consumption discount rate. ${ }^{3}$ Tol views the latter approach as the better approach. ${ }^{4}$ The latter approach is also the well-regarded view of Arrow et al. (1996) and is labelled by Gollier (2008) as the classical approach. In turn, Weikard and Zhu (2005) show formally in a model with a more general utility function than in the model in Tol (2004) that dual-rate discounting and the classical approach are equivalent. In particular, they show that use of dual-rate discounting requires future environmental benefits to be measured in constant relative prices of environmental quality. Further, in this case the environmental discount rate falls short of the consumption discount rate by the growth rate of the relative price of environmental quality. In contrast, the classical approach requires to adjust future environmental benefits for changes of the relative price of environmental quality and requires those benefits to be discounted at the consumption discount rate. It is fair to say that the classical approach is the most natural approach to pursue. ${ }^{5}$ In addition, after properly accounting for changes of the relative price of environmental quality, the classical approach can also justify substantial emission reductions.

Next, Hoel and Sterner (2007) complemented Tol (2004) with an alternative special case of the more general utility function of Weikard and Zhu (2005). More specifically, they assume each period's composite consumption index of environmental quality and goods consumption to be a CES function. Contrary to Tol (2004), Hoel and Sterner (2007) assume stable preference parameters, implying no direct effect of income growth on the WTP. However, they assume environmental resource scarcity in such a manner that growth of environmental quality falls short of growth of goods consumption. In their model, it is the environmental resource scarcity that leads to rising WTP and in turn this is reflected in a rising relative price of environmental quality, as in Weikard and Zhu (2005). Most importantly, Hoel and Sterner show that the growth rate of the relative price of environmental quality is the larger the lower the elasticity of substitution between environmental quality and goods consumption in each period's composite consumption index. Furthermore, building on the latter work, Sterner and Persson (2008) show that a rising relative price of environmental quality can justify the drastic level of emission reductions, recommended in the Stern Review. In particular, it can do so without reliance on ethical considerations, suggested in the Stern Review, which has been criticised so heavily by many economists as being paternalistic.

Finally, Traeger (2007) and Gollier (2008) show, using the same framework as Hoel and Sterner (2007), that the consumption discount rate and the environmental discount rate are both only time-invariant and unchanged from environmental resource scarcity if

\footnotetext{
${ }^{3}$ Richard Tol indirectly suggested this implication of his 2004 paper in an online-comment on an earlier version of my paper.

${ }^{4}$ Richard Tol expressed his preference for this approach in his aforementioned online-comment. A related article that promotes this approach is Horowitz (2002).

${ }^{5}$ Note however that according to Gollier (2008) in a framework with uncertainty, not applied to the present paper, dual-rate discounting has important advantages over the classical approach. In addition, in another online-comment on an earlier version of my paper, Reyer Gerlagh comments that only assuming a lower utility discount rate for environmental quality than for goods consumption gives enough credit to what the "inventors" of dual-rate discounting, Yang (2003) and Hasselmann et al. (1997), actually had in mind. As mentioned before, Yang's assumption requires use of dual-rate discounting.
} 
particular knife-edge parameter constellations are fulfilled. ${ }^{6}$ As Traeger (2007) stresses, this finding is important because the classical approach, as outlined by Arrow et al. (1996, p. 139), assumes a time-invariant consumption discount rate or at least that this discount rate is unchanged from environmental effects. As a consequence, recent research shows that it is not in general enough to adjust future environmental benefits for changes of the relative price of environmental quality. Instead, unless particular knife-edge parameter constellations are fulfilled, rising environmental scarcity requires in addition to adjust the consumption discount rate. ${ }^{7}$ The contribution of the present paper is to clarify that the aforementioned particular knife-edge parameter constellations are closely related to the concept of substitutability in the Edgeworth-Pareto sense (substitutability in the Edgeworth-Pareto sense describes the change of the marginal utility of goods consumption from increasing environmental quality). By implication, the present paper also challenges the view of Neumayer (1999), who argues that the discount rate debate regarding optimal climate change policy would miss the point, as substitutability rather than discounting would be the real issue. In contrast to Neumayer's argument, the present paper shows that the degree of substitutability in the Edgeworth-Pareto sense affects the time path of the consumption discount rate and therefore substitutability and discounting are issues that are closely linked.

Section 2 of the present paper reviews the equivalence between dual-rate discounting and the classical approach along the lines of Weikard and Zhu (2005). Next, section 3 introduces a utility function along the lines of Hoel and Sterner (2007), and derives in that framework a definition of substitutability in the Edgeworth-Pareto sense. Finally, section 4 derives the implication of substitutability in the Edgeworth-Pareto sense for the change of the discount rates over time, while section 5 concludes.

\section{Dual-Rate Discounting and the Classical Approach of Discounting}

Suppose a project of carbon emission reductions leads to reductions in goods consumption by $\Delta \mathrm{C}(\mathrm{t}), \forall \mathrm{t} \in[0, \infty]$, and to improvements of environmental quality by $\Delta \mathrm{E}(\mathrm{t}), \quad \forall \mathrm{t} \in[0, \infty]$. Equivalence between use of dual-rate discounting and use of the classical approach implies equivalence of the following two equations: 8

$$
N P V=\int_{0}^{\infty} \Delta \mathrm{C}(\mathrm{t}) e^{-R_{c}(t)} d t+p(0) \int_{0}^{\infty} \Delta \mathrm{E}(\mathrm{t}) e^{-R_{E}(t)} d t
$$

\footnotetext{
${ }^{6}$ Further, also using the framework as in Hoel and Sterner (2007) and adding uncertainty to it, Guesnerie (2004) argues that a plausible long-run value for the environmental discount rate is close to zero. In addition, it is fair to mention that a feedback effect from environmental resource scarcity to the discount rates had already been recognised in Hoel and Sterner (2007) itself and has been confirmed in that paper in numerical simulations.

${ }^{7}$ As a matter of fact, the aforementioned article by Sterner and Persson (2008) is a role model that shows how one should properly adjust in the classical approach both, future environmental benefits and the consumption discount rate, for environmental resource scarcity.

${ }^{8}$ See similar Weikard and Zhu (2005, pp. 874-875) for the case of time-invariant discount rates. Further, accounting for time-variant discount rates follows Groom et al. (2005, p. 453).
} 


$$
N P V=\int_{0}^{\infty} \Delta \mathrm{C}(\mathrm{t}) e^{-R_{c}(t)} d t+\int_{0}^{\infty} \mathrm{p}(\mathrm{t}) \Delta \mathrm{E}(\mathrm{t}) e^{-R_{c}(t)} d t
$$

with $R_{C}(t) \equiv \int_{0}^{t} r_{c}(s) d s$ and $R_{E}(t) \equiv \int_{0}^{t} r_{E}(s) d s$

where NPV denotes the present value of carbon emission reductions and $r_{C}(t)$ and $r_{E}(t)$ represent the consumption discount rate and the environmental discount rate. In addition, the price of consumption goods is normalised to one and $p(t)$ denotes the relative price of environmental quality. In (1), using dual-rate discounting, the NPV is calculated with the consumption discount rate, $r_{C}(t)$, and the environmental discount rate, $r_{E}(t)$. Further, in that equation future environmental benefits are measured in constant relative prices of environmental quality. Admittedly, this measure is somewhat unusual. In (2), using the classical approach, the NPV is calculated with the consumption discount rate, $r_{C}(t)$, properly accounting future environmental benefits for changes of the relative price of environmental quality. Using either (1) or (2), the decision maker should choose the emission reduction project that maximises the NPV. ${ }^{9}$

$$
g_{p}(t)=r_{c}(t)-r_{E}(t) \text { or } r_{E}(t)=r_{c}(t)-g_{p}(t)
$$

In turn, integrating the first equation in (3) and using the definitions of $R_{C}(t)$ and $R_{E}(t)$ in (1) and (2) yields:

$$
p(t) \equiv p(0) e^{\int_{0}^{t} g_{p}(s) d s}=p(0) e^{\int_{0}^{t} r_{C}(s) d s-\int_{0}^{t} r_{E}(s) d s}=p(0) e^{R_{c}(t)-R_{E}(t)} .
$$

Obviously, substituting (4) into (2) gives (1). This shows that (1) and (2) (and therefore dual-rate discounting and the classical approach) are equivalent. ${ }^{10}$ As mentioned in the introduction, it is fair to say that the classical approach is the most natural approach to pursue, as promoted by Arrow et al. (1996) and apparently preferred as well by Tol (2004). However, as has also been mentioned before, the classical approach usually imposes into (2) the assumption of a time-invariant consumption discount rate. In turn, as section 4 of the present paper will show, assuming environmental resource scarcity, a time-invariant consumption discount rate is, by and large, only appropriate in the knife-edge case in which environmental quality and goods consumption are neither substitutes nor complements in the Edgeworth-Pareto sense.

\footnotetext{
${ }^{9}$ Throughout the paper $g_{x}(t)$ denotes the growth rate of any variable $\mathrm{x}(\mathrm{t})$.

${ }^{10}$ See related Fisher and Krutilla (1975) and Perman et al. (2003, pp. 375-378).
} 


\section{Edgeworth-Pareto Substitutability}

Suppose the following life-time utility of a representative household:

$$
W(t)=\int_{0}^{\infty} U(\tilde{C}(t)) e^{-\rho t} d t
$$

where $U(\tilde{C}(t))$ represents instantaneous utility, $\tilde{C}(t)$ represents a composite consumption index of environmental quality and goods consumption and $\rho$ denotes the utility discount rate. In (5), instantaneous utility of different periods is assumed to be additively separable. Further, population growth is assumed to be absent.

Moreover, suppose instantaneous utility is defined as taking the following iso-elastic form:

$$
U(\tilde{C}(t))=\frac{\tilde{C}(t)^{1-\frac{1}{\varepsilon}}}{1-\frac{1}{\varepsilon}}
$$

where $\varepsilon$ denotes the intertemporal elasticity of substitution (which is the inverse of the elasticity of marginal utility with respect to the composite consumption index or the inverse of the coefficient of relative risk aversion).

Finally, suppose, along the lines of Hoel and Sterner (2007), the composite consumption index to take the following CES form:

$$
\tilde{C}(t)=\left[(1-\gamma) C(t)^{\frac{\sigma-1}{\sigma}}+\gamma E(t)^{\frac{\sigma-1}{\sigma}}\right]^{\frac{\sigma}{\sigma-1}}, \text { with } \sigma \geq 0
$$

In (7), $\sigma$ is the intertemporal elasticity of substitution between environmental quality and goods consumption and $\gamma$ is a constant parameter.

In addition, after substituting (7) in (6), it can be shown that environmental quality and goods consumption are neither substitutes nor complements in the EdgeworthPareto sense if: 11

$$
\eta_{C E}(t)=\gamma^{*}(t)\left(\frac{1}{\sigma}-\frac{1}{\varepsilon}\right)=0
$$

with $\eta_{C E}(t) \equiv \frac{E(t) U_{C E}(t)}{U_{C}(t)}$ and $\gamma^{*}(t) \equiv \frac{\gamma E(t)^{\frac{\sigma-1}{\sigma}}}{(1-\gamma) C(t)^{\frac{\sigma-1}{\sigma}}+\gamma E(t)^{\frac{\sigma-1}{\sigma}}}$,

where $U_{C}(t) \equiv \partial U(t) / \partial C(t)$ and $U_{C E}(t) \equiv \partial^{2} U(t) / \partial C(t) \partial E(t)$. Further, $\gamma^{*}(t)$ denotes the value share of environmental goods (see Gerlagh and van der Zwaan, 2002, and Hoel and Sterner 2007). In contrast, as Table 1 shows, environmental quality and goods consumption are substitutes in the Edgeworth-Pareto sense if $\eta_{C E}(t)<0$ and complements in the Edgeworth-Pareto sense if $\eta_{C E}(t)>0$.

${ }^{11}$ See, e.g., Samuelson (1974, page 1256, footnote 2). Related, see also Amano and Wirjanto (1998). 
Table 1. Cases of Edgeworth-Pareto (EP) Substitutability.

\begin{tabular}{|l|c|c|c|}
\hline & $\begin{array}{c}(1 / \sigma)=(1 / \varepsilon) \\
\Rightarrow \eta_{C E}(t)=0\end{array}$ & $\begin{array}{c}(1 / \sigma)<(1 / \varepsilon) \\
\Rightarrow \eta_{C E}(t)<0\end{array}$ & $\begin{array}{c}(1 / \sigma)>(1 / \varepsilon) \\
\Rightarrow \eta_{C E}(t)>0 .\end{array}$ \\
\hline EP substitutability & neutral & substitutes & complements \\
\hline
\end{tabular}

It is straightforward to verify, after substituting (7) in (6), that fulfilment of $\eta_{C E}(t)=0$ and therefore $(1 / \sigma)=(1 / \varepsilon)$ (cf. Table 1$)$ implies :

$$
\mathrm{U}(\mathrm{t})=(1-\gamma) \frac{\mathrm{C}(\mathrm{t})^{1-\frac{1}{\sigma}}}{1-\frac{1}{\sigma}}+\gamma \frac{\mathrm{E}(\mathrm{t})^{1-\frac{1}{\sigma}}}{1-\frac{1}{\sigma}}
$$

where the instantaneous utility function is in this case additively separable in environmental quality and goods consumption.

\section{Change of Discount Rates over Time}

So far we did not derive the determinants of the discount rates. Indeed, it is tempting to assume them to be time-invariant. However, as mentioned in the introduction, Traeger (2007) and Gollier (2008) show, using more or less the framework of the present paper's last section, that the consumption discount rate and the environmental discount rate are both only time-invariant under particular knife-edge parameter constellations. This section clarifies that these particular knife-edge parameter constellations are closely related to the concept of substitutability in the Edgeworth-Pareto sense.

The starting point of analysis is to derive the discount rates from a social planner's optimisation problem. Combining (5)-(7), the social planner maximises:

$$
W(t)=\left[1 /\left(1-\frac{1}{\varepsilon}\right)\right] \int_{0}^{\infty}\left[(1-\gamma) C(t)^{\frac{\sigma-1}{\sigma}}+\gamma E(t)^{\frac{\sigma-1}{\sigma}}\right]^{\left(\frac{\sigma}{\sigma-1}\right)\left(1-\frac{1}{\varepsilon}\right)} e^{-\rho t} d t
$$

subject to the budget constraint:

$$
\int_{0}^{\infty} C(t) e^{-R_{c}(t)} d t+\int_{0}^{\infty} p(t) E(t) e^{-R_{c}(t)} d t=Y
$$

where $Y$ represents the present value of resources available for consumption of environmental quality and consumption goods over the representative household's lifetime. ${ }^{12}$

Setting a Lagrangian and using (7) gives the following first order conditions: ${ }^{13}$

\footnotetext{
${ }^{12}$ See similar in Creedy (2007).

${ }^{13}$ Note that in the derivations use is made of the fact that [.] $]^{\left(\frac{\sigma}{\sigma-1}\right)\left(1-\frac{1}{\varepsilon}\right)-1}=\widetilde{\mathrm{C}}(\mathrm{t})^{\left(\left[\frac{\sigma}{\sigma-1}\right)\left(1-\frac{1}{\varepsilon}\right)-1\right]\left[\frac{\sigma-1}{\sigma}\right]}$ (cf. eq. (7)) and hence [.] $]^{\left(\frac{\sigma}{\sigma-1}\right)\left(1-\frac{1}{\varepsilon}\right)-1}=\tilde{\mathrm{C}}(\mathrm{t})^{\frac{\varepsilon-1}{\varepsilon}-\frac{\sigma-1}{\sigma}}=\tilde{\mathrm{C}}(\mathrm{t})^{\frac{1}{\sigma}-\frac{1}{\varepsilon}}$. In addition, $\lambda$ denotes a constant Lagrangian multiplier.
} 


$$
\begin{gathered}
(1-\gamma) C(t)^{-\frac{1}{\sigma}} \tilde{C}(t)^{\frac{1}{\sigma}-\frac{1}{\varepsilon}} e^{-\rho t}=\lambda e^{-R_{c}(t)}, \\
\gamma E(t)^{-\frac{1}{\sigma}} \tilde{C}(t)^{\frac{1}{\sigma}-\frac{1}{\varepsilon}} e^{-\rho t}=\lambda p(t) e^{-R_{c}(t)} .
\end{gathered}
$$

Taking natural logarithms of (9) and (10), differentiating the resulting expressions with respect to time, using the fact that $d R_{C}(t) / d t=r_{C}(t)$ and rearranging terms yields:

$$
\begin{aligned}
r_{C}(t)=\rho+\frac{1}{\sigma} g_{C}(t)-\left(\frac{1}{\sigma}-\frac{1}{\varepsilon}\right) g_{\tilde{C}}(t), \\
r_{C}(t)=\rho+\frac{1}{\sigma} g_{E}(t)-\left(\frac{1}{\sigma}-\frac{1}{\varepsilon}\right) g_{\tilde{C}}(t)+g_{p}(t), \\
\text { with } \quad g_{\tilde{C}}(t)=\left(1-\gamma^{*}(t)\right) g_{C}(t)+\gamma^{*}(t) g_{E}(t) .
\end{aligned}
$$

Note that (13) shows the growth rate of the composite consumption index, $g_{\tilde{C}}(t)$, to be a weighted average of the growth rates of goods consumption and environment quality, weighted by their respective value shares. ${ }^{14}$

Next, substituting (3) for $g_{p}(t)$ into (12) and rearranging terms gives the environmental discount rate as:

$$
r_{E}(t)=\rho+\frac{1}{\sigma} g_{E}(t)-\left(\frac{1}{\sigma}-\frac{1}{\varepsilon}\right) g_{\tilde{C}}(t) .
$$

Finally, upon use of the definition of $\gamma(t)^{*}$ in (8), taking natural logarithms and differentiating the resulting expressions within respect to time, it can easily be shown that:

$$
\begin{gathered}
g_{1-\gamma^{*}}(t)=-\left(\frac{1-\sigma}{\sigma}\right) \gamma^{*}(t)\left(g_{C}(t)-g_{E}(t)\right), \\
g_{\gamma^{*}}(t)=\left(\frac{1-\sigma}{\sigma}\right)\left(1-\gamma^{*}(t)\right)\left(g_{C}(t)-g_{E}(t)\right) .
\end{gathered}
$$

Suppose now that there is environmental resource scarcity in such a manner that $g_{C}(t)>g_{E}(t)$ (as is assumed in Hoel and Sterner (2007) and as seems reasonable). We can then distinguish between the following two cases:

14 Note also that if we impose $(1 / \sigma)=(1 / \varepsilon)$ (i.e. there to be neither substitutability nor complementarity in the Edgeworth-Pareto sense - cf. Table 1), then eq. (11) reduces to the standard Keynes-Ramsey rule in optimal growth models. 
Case 1: $\sigma<1$ (that is, the elasticity of substitution between environmental quality and goods consumption in $\tilde{C}(t)$ is smaller than one)

As is also shown in Gerlagh and van der Zwaan (2002), $g_{C}(t)>g_{E}(t)$ implies according to (15) and (16) the value share of goods consumption, $1-\gamma(t)^{*}$, to decline over time and the value share of environmental quality, $\gamma(t)^{*}$, to rise over time. In turn, in (13) this implies a declining weight of $g_{C}(t)$ and a rising weight of $g_{E}(t)$ in growth of the composite consumption index, $g_{\tilde{C}}(t)$. Since $g_{C}(t)>g_{E}(t)$, this implies that $g_{\tilde{C}}(t)$ declines over time. As a consequence, (11) and (14) imply over time declining discount rates if $(1 / \sigma)<(1 / \varepsilon)$, that is, if environmental quality and goods consumption are substitutes in the Edgeworth-Pareto sense (cf. Table 1). In contrast, if $(1 / \sigma)>(1 / \varepsilon)$, that is, if environmental quality and goods consumption are complements in the Edgeworth-Pareto sense (cf. Table 1), then both discount rates rise over time.

Case 2: $\sigma>1$ (that is, the elasticity of substitution between environmental quality and goods consumption in $\tilde{C}(t)$ is larger than one)

In this case, according to (15) and (16), $g_{C}(t)>g_{E}(t)$ implies $1-\gamma(t)^{*}$ to rise over time and $\gamma(t)^{\star}$ to decline over time (cf. Gerlagh and van der Zwaan (2002)). Therefore, in (13) the weight of $g_{C}(t)$ rises, while the weight of $g_{E}(t)$ declines. As a result of this, $g_{C}(t)>g_{E}(t)$ implies now $g_{\tilde{C}}(t)$ to rise over time. Hence, now (11) and (14) imply rising discount rates if $(1 / \sigma)<(1 / \varepsilon)$ (i.e. if we have substitutes in the EdgeworthPareto sense), while both discount rates decline if $(1 / \sigma)>(1 / \varepsilon)$ (i.e. if we have complements in the Edgeworth-Pareto sense).

Most importantly, eq. (11) and (14) show that, provided $\sigma \neq 1$, then time- invariance of both discount rates, as usually assumed in the classical approach, requires $(1 / \sigma)=(1 / \varepsilon)$, that is, requires environmental quality and goods consumption to be neither substitutes nor complements in the Edgeworth-Pareto sense. If they are substitutes or complements in the Edgeworth-Pareto sense, then the only alternative condition that ensures time-invariance of both discount rates is $\sigma=1$ (as in this case (15) and (16) imply the value shares of goods consumption and environmental quality to be constant and therefore, according to $(13), g_{\tilde{C}}(t)$ to be time-invariant, which implies according to (11) and (14) time-invariant discount rates).

Further, a note on Traeger (2007) is necessary. In contrast to the present paper, Traeger argues that over time declining discount rates requires $\sigma>1$. However, as the present framework shows, this result is only due to the fact that he assumes in his model $(1 / \varepsilon)=0$. Imposing $(1 / \varepsilon)=0$ in the equation describing substitutability in the Edgeworth-Pareto sense, i.e. in $\eta_{C E}(t)=\gamma^{*}(t)[(1 / \sigma)-(1 / \varepsilon)]$, implies $\eta_{C E}(t)>0$ if $\sigma>0$ (i.e. that we have complements in the Edgeworth-Pareto sense if $\sigma>0$ ). In turn, the abovementioned case 2 (i.e. $\sigma>1$ ) implied declining discount rates in case of complements in the Edgeworth-Pareto sense (see the discussion above). As in case 
$\sigma>1$ the discount rates also decline in Traeger (2007), his result is in line with the present paper's result, though Traeger's result is only a special case of the present paper's result. Note however that $(1 / \varepsilon)$ represents the coefficient of relative risk aversion. Therefore, the assumption $(1 / \varepsilon)=0$ implies risk neutrality. However, it seems more realistic to assume the representative household to be risk averse. Risk aversion in turn implies $(1 / \varepsilon)>0$. As the present study showed, in this case matters are more complicated and therefore introduction of the concept of substitutability in the Edgeworth-Pareto sense turns out to be convenient.

Finally, also a note on Neumayer (1999) is useful. Neumayer argues that substitutability rather than discounting is the real issue for optimal climate change policy. In contrast to Neumayer's argument, the present paper shows that the degree of substitutability affects the discount rates and therefore substitutability and discounting are issues that are closely linked. In particular, the aforementioned case 1 shows that $\sigma<1$ (that is, an elasticity of substitution between environmental quality and goods consumption in $\tilde{C}(t)$ that is smaller than one) together with $(1 / \sigma)<(1 / \varepsilon)$ and therefore $\varepsilon<\sigma$ (that is, an even lower intertemporal elasticity of substitution) imply over time declining discount rates.

\section{Conclusion}

To justify substantial carbon emission reductions, recent work introduced dual-rate discounting into the literature of cost-benefit analysis of climate change. Yang (2003) justifies discounting environmental quality at a low environmental discount rate by assuming different utility discount rates for environmental quality and goods consumption (the former being lower than the latter). Yang's assumption of different utility discount rates has however been criticised by Tol (2004) as being an ad hoc assumption that lacks theoretical foundation. Nevertheless, maintaining the conventional assumption of a single utility discount rate for environmental quality and goods consumption, Hoel and Sterner (2007) show that a low environmental discount rate can be justified by assuming environmental resource scarcity. As the authors show, environmental resource scarcity leads to a growing relative price of environmental quality. Further, as already shown by Weikard and Zhu (2005), the environmental discount rate falls short of the consumption discount rate by the growth rate of the relative price of environmental quality. As a consequence, environmental resource scarcity can justify a low environmental discount rate. Despite of this, Tol (2004) appears to object dual-rate discounting as to be confusing and unnecessary. Instead, he appears to prefer the classical approach, which requires to properly account future environmental benefits for changes of the relative price of environmental quality and requires then to discount those benefits at the consumption discount rate. Indeed, it is fair to say that the classical approach is the most natural approach to pursue. However, as has been argued by Traeger (2007), the classical approach usually assumes a timeinvariant discount rate. As Traeger (2007) and Gollier (2008) show, the framework of Hoel and Sterner (2007) does not generally imply a time-invariant consumption discount rate. Instead, time-variance of the consumption discount rate requires fulfilment of particular knife-edge parameter constellations. For this reason, it is not in 
general enough to adjust future environmental benefits for environmental effects. The present paper clarified that the aforementioned particular knife-edge parameter constellations imply, by and large, environmental quality and goods consumption to be neither substitutes nor complements in the Edgeworth-Pareto sense (where the degree of substitutability in the Edgeworth-Pareto sense measures the change of the marginal utility of goods consumption from increasing environmental quality). Even if one believes this knife-edge property to be approximately fulfilled in reality, it is important to be aware that such a knife-edge property is imposed.

\section{Appendix: Derivation of Eq. (3)}

As mentioned in the text, efficient allocation requires the relative price of environmental quality to equal the marginal rate of substitution between environmental quality and goods consumption, that is, efficiency requires $p(t)=U_{E}(t) / U_{C}(t)$. In turn, after substituting (7) in (6), the latter can be shown to imply:

$$
p(t)=\left(\frac{\gamma}{1-\gamma}\right)\left(\frac{C(t)}{E(t)}\right)^{\frac{1}{\sigma}} .
$$

Next, taking natural logarithms of (A.1) and differentiating the resulting expression with respect to time yields:

$$
g_{p}(t)=\frac{1}{\sigma}\left(g_{C}(t)-g_{E}(t)\right) .
$$

Furthermore, combining (11) and (14) implies:

$$
r_{c}(t)-r_{E}(t)=\frac{1}{\sigma}\left(g_{C}(t)-g_{E}(t)\right) .
$$

Obviously, combining (A.2) and (A.3) gives rise to eq. (3) in the text. 


\section{References}

Amano R.A., and T.S. Wirjanto (1998). Government Expenditures and the Permanent-Income Model. Review of Economic Dynamics 3: 719-730.

Arrow, K., Cline, W., Mäler, K.-G., Munasinghe, M., Squitieri, R., and J. Stiglitz (1996). Intertemporal Equity, Discounting, and Economic Efficiency. In J. Bruce, H. Lee, and E. Haites (eds.) Climate Change 1995 - Economic and Social Dimensions of Climate Change. Cambridge: Cambridge University Press.

Creedy, J. (2007). Discounting and the Social Time Preference Rate. University of Melbourne Department of Economics Working Paper.

Fisher, A.C. and J.V. Krutilla, (1975). Resource Conservation, Environmental Preservation, and the Rate of Discount. Quarterly Journal of Economics 89: 358-370.

Gerlagh, R., and B.C.C. van der Zwaan (2002), Long-term Substitutability between Environmental and Man-Made Goods. Journal of Environmental Economics and Management 44: 329-345.

Gollier, C. (2008). Ecological Discounting. Paper presented at the EAERE Annual Conference 2008 in Gothenburg, Sweden.

Groom, B., Hepburn, C., Koundouri, P., and D. Pearce (2005). Declining Discount Rates: The Long and the Short of it. Environmental and Resource Economics 32: 445-493.

Guesnerie, R. (2004). Calcul economique et developpement durable. La Revue Economique 55: $363-382$.

Hasselmann K, Hasselmann S., Giering R., Ocana V., and H. von Storch (1997). Sensitivity Study of Optimal CO2 Emission Paths Using a Simplified Structural Integrated Assessment Model. Climatic Change 37: 345-386.

Hoel M. and T. Sterner (2007). Discounting and Relative Prices. Climatic Change 84: 15731480 .

Horowitz, J.K. (2002). Preferences in the Future. Environmental and Resource Economics 21: 241-259.

Kaldor, N. (1961). Capital Accumulation and Economic Growth. In F.A. Lutz and D.C. Hague (eds.), The Theory of Capital. New York: St. Martins.

Neumayer, E. (1999). Global Warming: Discounting is not the Issue, but Substitutability is. Energy Policy 27: 33-43.

Nordhaus, W.D. (2007). A Review of the Stern Review on the Economics of Climate Change. Journal of Economic Literature 45: 686-702.

Nordhaus,W.D., and J. Boyer (2000). Warming the World. Cambridge, MA: MIT Press.

Nordhaus, W.D., and Z. Yang (1996). A Regional Dynamic General-Equilibrium Model of Alternative Climate-Change Strategies. American Economic Review 86: 741-765.

Perman, R., Ma, Y., McGilvray, J., and M. Common (2003). Natural Resource and Environmental Economics. $3^{\text {rd }}$ edition. Harlow, England: Pearson/ Addison Wesley.

Samuelson, P. (1974). Complementarity: An Essay on the 40th Anniversary of the Hicks-Allen Revolution in Demand Theory. Journal of Economic Literature 12: 1255-1289. 
Stern, N., Peters, S., Bakhshi, V., Bowen, A., Cameron, C., Catovsky, S., Crane, D., Cruickshank S., Dietz, S., Edmonson, N., Garbett, S.-L., Hamid, L., Hoffman, G., Ingram, D., Jones, B., Patmore, N., Radcliffe, H., Sathiyarajah, R., Stock, M., Taylor, C., Vernon, T., Wanjie, H., and D. Zenghelis (2006). Stern Review: The Economics of Climate Change, London: HM Treasury.

Sterner, T., and U.M. Persson (2008). An Even Sterner Review: Introducing Relative Prices into the Discounting Debate. Review of Environmental Economics and Policy 2: 61-76.

Tol, R.S.J. (2004). On Dual-Rate Discounting. Economic Modelling 21: 95-98.

Traeger, C. (2007). Sustainability, Limited Substitutability and Non-Constant Social Discount Rates. Department of Agriculture and Resource Economics Discussion Paper 1045, University of California, Berkeley.

Weikard H.-P., and X. Zhu (2005). Discounting and Environmental Quality: When should Dual Rates be used? Economic Modelling 22: 868-878.

Weitzman, M. (2007). The Stern Review of the Economics of Climate Change. Journal of Economic Literature 45: 703-724.

Yang, Z. (2003). Dual-Rate Discounting in Dynamic Economic-Environmental Modelling. Economic Modelling 20: 941-957.

Yohe, G.W., and R.S.J. Tol (2007). The Stern Review: Implications for Climate Change. Environment 49: 36-42. 


\section{conomics}

The Open-Access, Open-Assessment E-Journal

\section{Please note:}

You are most sincerely encouraged to participate in the open assessment of this article. You can do so by either rating the article on a scale from 1 (bad) to 5 (excellent) or by posting your comments.

Please go to:

www.economics-ejournal.org/economics/journalarticles/2009-27

The Editor 